\title{
Measurement of Cyclic Flows in Trachea Using PIV and Numerical simulation
}

\author{
Miloslav Bělka ${ }^{1, a}, J$ akub Elcner ${ }^{1}$ Jan Jedelský $^{1}$, Olivier Boiron ${ }^{2}$,Yannick Knapp ${ }^{3}$ and Lucie Bailly ${ }^{2}$ \\ ${ }^{1}$ Brno University of Technology, Technická 2896/2, 61669 Brno, Czech Republic \\ ${ }^{2}$ Aix Marseille Université, CNRS, Centrale Marseille, IRPHE UMR 7342, F-13384, Marseille, France \\ ${ }^{3}$ Avignon University, LAPEC EA4278, F-84000, Avignon, France
}

\begin{abstract}
Inhalation of pharmaceutical aerosols is a convenient way to treat lung or even systemic diseases. For effective treatment it is very important to understand air flow characteristics within respiratory airways and determine deposition hot spots. In this paper the air flow in trachea was investigated by numerical simulations. To validate these results we carried out particle image velocimetry experiments and compared resulting velocity fields. Simplified geometry of respiratory airways from oral cavity to 4 th generation of branching was employed. Air flow characteristics were analysed during sinusoidal breathing pattern for light activity conditions (period $4 \mathrm{~s}$ and tidal volume 11 ). The observed flow fields indicated that the flow in trachea is turbulent during the sinusoidal flow except phases of flow turnarounds. The flow was skewed to front side of the trachea during inspiration and had twin-peak profile during expiration because of the mixing from daughter branches. The methods were compared and good agreement was found. This validation of CFD simulation can result into its further usage in respiratory airflow studies.
\end{abstract}

\section{Introduction}

The amount of people, who suffer from lung diseases, such as asthma or chronic obstructive pulmonary disease (COPD), increases every year. Target delivery of pharmaceutical aerosols is vital for appropriate treatment of these diseases. For effective treatment it is very important to understand the deposition patterns, which are influenced by both particle and air flow characteristics.

The air flow in respiratory airways is very complex because of the intricacy of their geometry. For example glottis and other complicated larynx structures influence air flow in trachea and create so-called laryngeal jet [1]. This laryngeal jet impinges air flow towards the wall of the trachea and creates areas of increased deposition. Many studies dealt with analysis of flow characteristics, but only few of them used realistic models in their experiments. Unfortunately this is essential to obtain correct results [2].

Two approaches are employed to study air flow in respiratory airways, in vitro experiments and computational fluid dynamics (CFD) simulations. Even though the CFD provides simplicity in using realistic models and conditions, potential input errors can lead to non-realistic results. From this point of view it is very important to validate CFD simulations experimentally before performing more complex studies [3].
In experiments optical methods are very frequent, but their requirement for optical access often results in simplified conditions with semirealistic models. Ramuzat et al [4] used symmetrical model of several generations of branching and studied air flow within bifurcation. Grosse et al [5] executed experiments with realistic silicone model and analysed secondary vortices around first bifurcation. Kim et al [6] obtained particle image velocimetry (PIV) results from realistic model with realistic conditions. They observed the main stream of air at the backside of trachea during inspiration and at the frontal side during expiration, which prevents mixing of the fresh air. Almost all of the studies substituted air with another working fluid, such as water with glycerine, to avoid optical distortions.

CFD simulations in extrathoric region were performed by Johnstone et al [7], although their geometry did not include bronchial tree. Zhang \& Kleinstreuer investigated flow characteristics and deposition in simplified model, which consisted of nasal and oral cavity, larynx, trachea and three generations of branching using Menters SST model [8]. Lin et al [9] analyzed air flow in larynx and trachea in realistic model of upper respiratory airways including branching up to 7 th generation.

The aim of this paper was to carry out in vitro experiments and CFD simulations and to make a comparison. The same geometry of semirealistic model

\footnotetext{
a Belka@fme.vutbr.cz
} 
of upper respiratory airways was used in both methods. The results were compared and good agreement was found. These validations can lead to a further study of air flow in respiratory airways using CFD.

\section{$2 \quad$ Materials and methods}

Investigation of the air flow was performed in a simplified model of respiratory airways from oral cavity up to 4th generation of branching (Figure 1) by means of PIV and CFD simulations. For the purposes of the experiments the model consisted of a realistic oral cavity, pharynx, larynx and the conducting airways, which were substituted by thin-walled glass tubes. The geometry of conducting airways preserved real branch volumes, branch lengths and branching angles. The measurements were conducted in trachea above the first bifurcation (Figure 1) in different phases of sinusoidal cyclic flow for light activity conditions (period of $4 \mathrm{~s}$ and tidal volume of $11)$.
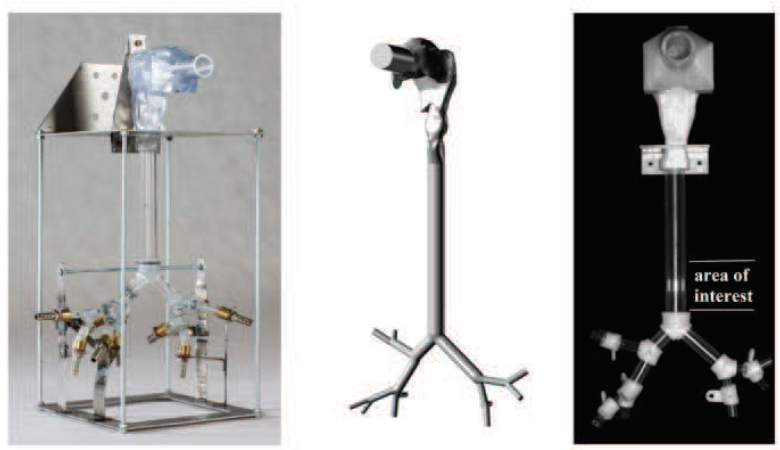

Figure 1. Experimental and CFD model of respiratory airways

\subsection{PIV measurements}

Particle Image Velocimetry is a common method for analysis of air flow characteristics and provides 2 component velocity field. The experimental setup (Figure 2) consisted of piston based breathing simulator, model of human lungs and PIV system. The flow was seeded with particles from incense sticks and the particles were delivered to piston chambers as well as to the outputs of the model to ascertain sufficient concentration during both inspiration and expiration.

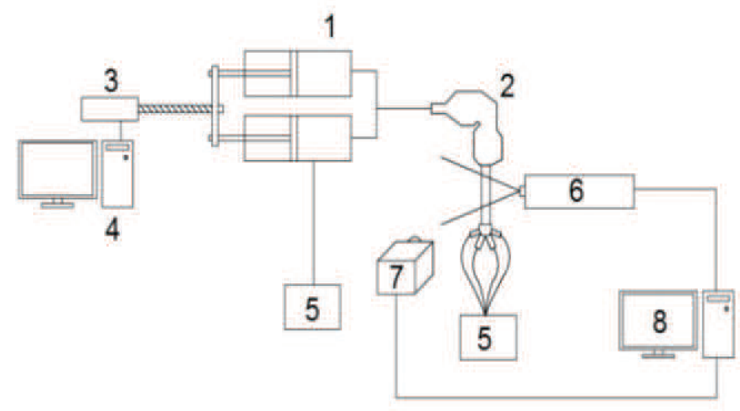

Figure 2. Schematics of the experimental setup. 1, breathing simulator; 2, lung model; 3 , servomotor; 4 , computer; 5 ,smoke generator ; 6 , pulse laser ; 7, PIV camera ; 8 , computer
Components of the PIV system were situated around the model to collect the data. A collimated light sheet approximately $1 \mathrm{~mm}$ thick, $10 \mathrm{~cm}$ large was produced by a dual-head pulsed Nd:YAG laser (New Wave Gemini, $120 \mathrm{~mJ}$ per pulse, repetition rate $4 \mathrm{~Hz}$ ). TSI power view $4 \mathrm{Mp}$ camera with a polarizing filter was situated perpendicularly to the light sheet to obtain images of the flow in the sagittal plane. Timing of all components was executed by TSI synchroniser and images were processed using Insight $3 \mathrm{G}$ software. Measurements of eight phases of sinusoidal cycle were carried out. A set of 80 images was captured for every phase setting to enable calculation of velocity field averages. Cross-correlation was performed to determine the displacement of the particles within a $50 \%$ overlapping interrogation window $32 \times 32$ pixel in size. Every velocity field passed a post-process validation and incorrect vectors were substituted by a median value from surrounding vectors.

\subsection{CFD simulations}

Mesh generation and numerical simulation were executed in software StarCCM+ version 8.02. The polyhedral mesh contained 2.6 million elements including prismatic cells near the walls for proper computing of boundary layers. The mesh was generated in order to fulfill low wall $\mathrm{Y}+$ treatment $(\mathrm{Y}+<1)$ and to get appropriate Courant number during high velocity phases of the flow according to the time step $0.001 \mathrm{~s}$.

The simulation was calculated for one breathing cycle (light activity conditions) and Reynolds averaged NavierStokes (uRANS) model was employed. Because the range of Reynolds number in a branching tree of respiratory airways is very large $\left(300-10^{4}\right)[10]$, Menter hybrid SST model of turbulence [11] with modification for low Reynolds number was applied.

Boundary conditions for both inlet and outlet were set to be in compliance with the experiment. Pressure outlet was used as the outlet boundary condition. The inlet boundary condition was prescribed at the end of oral cavity extension and was defined by equation 1 , which determines velocity for every phase of sinusoidal flow.

$$
v(t)=\frac{V_{t} \cdot \pi}{S \cdot T} \cdot \sin \left(\frac{2 \pi}{T} \cdot t\right) \quad\left(m \sec ^{-1}\right)
$$

where $v, V_{t}, S, T$ a $t$ are velocity, tidal volume, surface, period and time step respectively.

\subsection{Flow characteristics}

Dimensionless numbers, in particular Reynolds and Womersley number, were calculated based on the diameter of trachea and standard air properties. Reynolds number determines laminar or turbulent flow and the critical value is 2300 in straight tubes. It can be calculated with

$$
R e=\frac{v \cdot D}{v}
$$


Where $D$ is characteristic dimension (diameter) and $v$ is air kinematic viscosity. Womersley number quantifies unsteadiness of the flow and represents the ratio of unsteady inertial forces to viscous forces. It can be calculated using

$$
\alpha=\frac{L}{2} \cdot \sqrt{\frac{n}{v}}
$$

Where $L$ is characteristic dimension (diameter), $n$ is frequency of oscillation in radians. The flow can be considered quasisteady if the Womersley number is smaller than five [12].

\section{$3 \quad$ Results}

The general aim of this paper was to compare experimental and numerical results of velocity fields in trachea. Flow investigations were performed in eight phases of sinusoidal flow for both methods $(0,45,90, \ldots$ $360^{\circ}$ ), three phases for inspiration, three for expiration and two transitions (I-E, E-I) as can be seen in Figure 3.

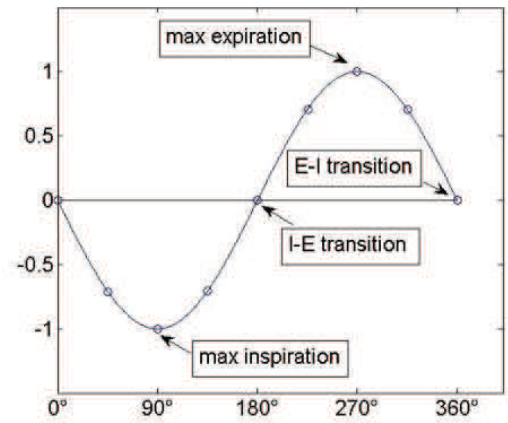

Figure 3. Measured sinusoidal pattern
Dimensionless numbers were calculated beforehand for every phase and can be found in Table 1 except numbers for transition phases, in which zero velocities were expected. The Womersley number 2.7 suggests quasisteady flow. Reynolds numbers for all phases were higher than critical Reynolds number for straight pipe flows, which indicates turbulent flow.

Table 1 Dimensionless numbers

\begin{tabular}{|c|c|c|}
\hline Phase (\%) & $\boldsymbol{R e}(-)$ & $\boldsymbol{\alpha}(-)$ \\
\hline $45,135,225,315$ & 3640 & 2.7 \\
\hline 90,270 & 5147 & 2.7 \\
\hline
\end{tabular}

Velocity fields of both methods at different selected instants are displayed in Figure 3. For clarity, only every second $x$ vector and seventh $y$ vector is displayed in the images. Velocity fields are skewed during inspiration, which was probably caused by the shape of glottis aperture creating so-called laryngeal jet. The maximum value of velocity was shifted to the front trachea wall. Mixing of the flow from daughter branches resulted in higher velocities near the walls during expiration. Visual comparison shows similar patterns for both experimental and numerical approaches.

Velocity profiles for $y=15 \mathrm{~mm}$ at eight measured flow phases are shown in Figure 5. The velocities were normalized, which was done by dividing them by the highest velocity value, i.e. peak expiration velocity. As can be seen the profiles are highly asymmetrical during inspiration with highest value skewed to the front of the trachea. Both methods agreed on this asymmetry. The inspiration profiles are flat-like suggesting turbulent flow which is in agreement with calculated Reynolds numbers. Twin-peak shapes of the flow were formed during expiration phases. It can be observed that the phases for the specific part of the sinusoidal flow (inspiration,
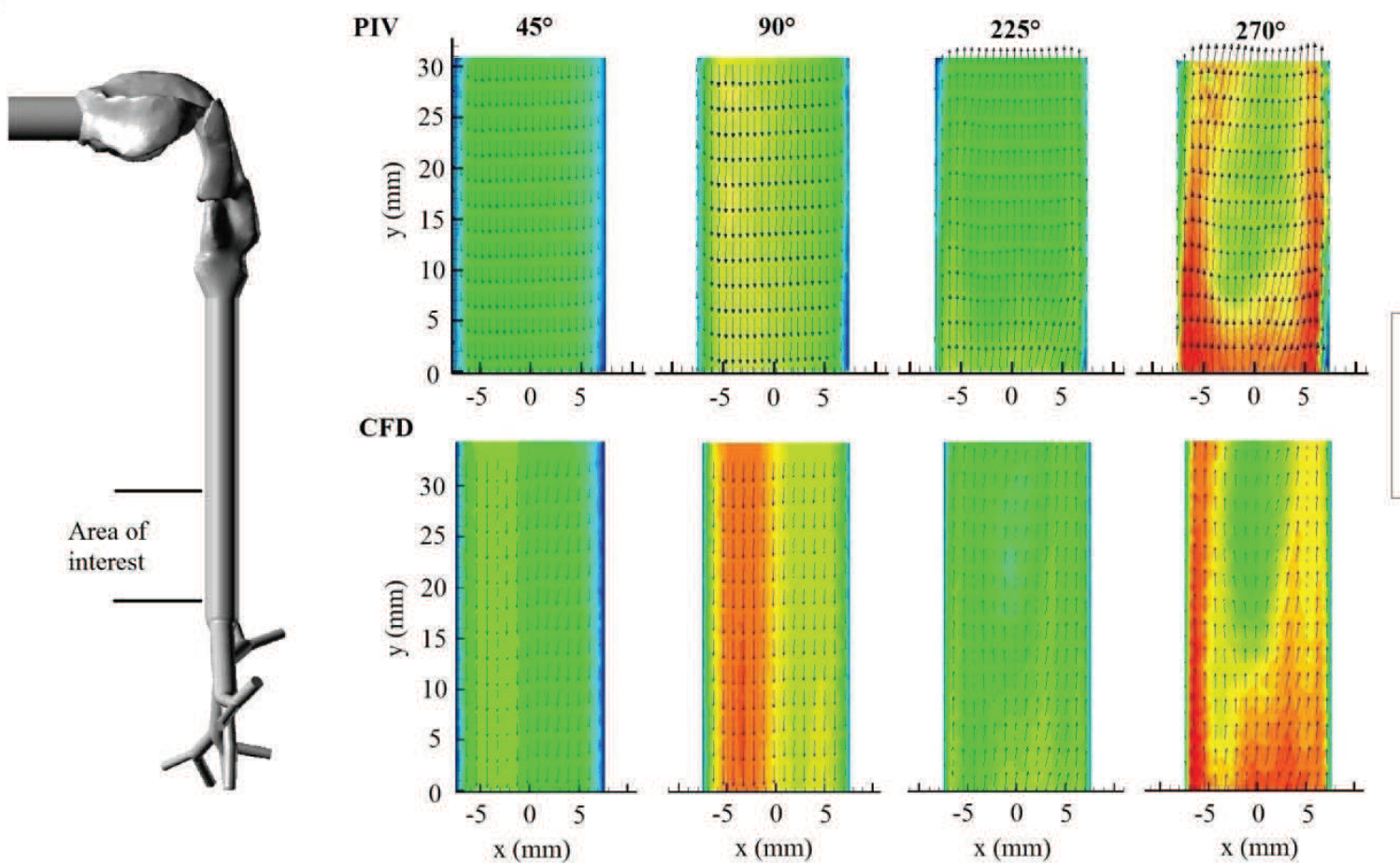

Figure 4. Side view of the model geometry; Comparison of velocity fields 
expiration) are very similar. After the turbulent flow was set up, increasing velocity only influences the amplitude of the profile.

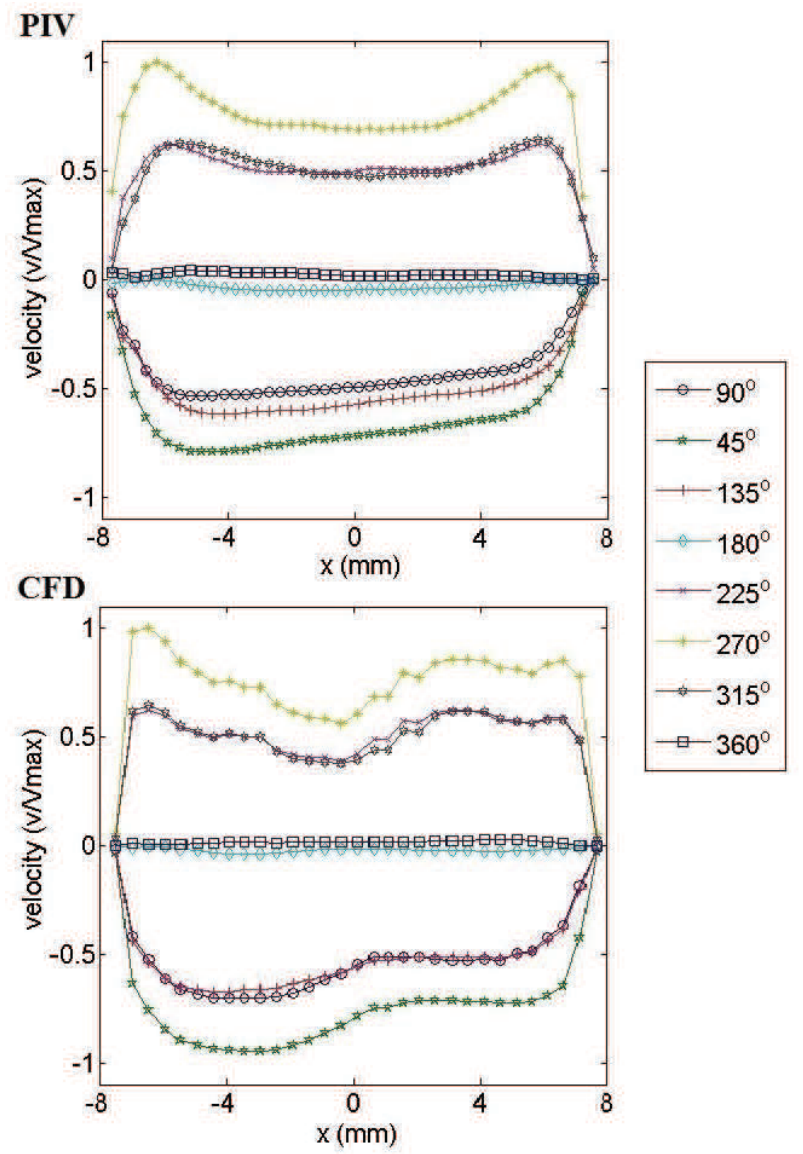

Figure 5. Averaged velocity profiles

The results from both methods show several physical phenomena which can increase deposition in trachea. First of all laryngeal jet, which impinges flow to the wall of the trachea and can cause increased deposition by impaction. Moreover turbulent flow during light activity breathing regime can also force some particles to impact on the walls.

\section{Conclusions}

CFD simulations are powerful and fast tool for investigation of fluid dynamics, but results from these methods need validation. We analysed air flow in human trachea using both in vitro experiments and CFD simulations and we find a good agreement. Both methods agreed on different physical phenomena, in particular laryngeal jet impinging the flow to the front of trachea or mixing of the flow during expiration causing highest velocities near the walls.

Several phases of sinusoidal breathing regime were measured employing PIV system and conditions of this regime simulated light activity. Calculated Womersley numbers suggested quasisteady flow, Reynolds number indicated turbulent flow in all phases except transition phases and this fact was confirmed by results from PIV and CFD. The velocity profiles were flat and skewed to the front side of trachea during inspiration. Twin-peak velocity profiles were observed during expiration.

It is worth noting that this is a first step in validation of our CFD. In future we will compare other regimes such as regime for sedentary breathing or heavy-activity conditions and we will analyse flow in main bronchi and further branching generations.

\section{Acknowledgement}

Authors acknowledge financial support from the joint Czech - French project MOBILITY № 7AMB13FR047 (Flow and transport of medical aerosols in the human airways) and from the project FCH/FSI-J-14-2479 (BD13402000), both funded by the Ministry of Education, Youth and Sports of the Czech Republic.

\section{References}

T. E. Corcoran and N. Chigier, Journal of Aerosol Medicine-Deposition Clearance and Effects in the Lung 13 (2), 125 (2000).

2 C. Kleinstreuer and Z. Zhang, Annual Review of Fluid Mechanics 42, 301 (2010).

3 C. van Ertbruggen, P. Corieri, R. Theunissen, M. Riethmuller, and C. Darquenne, Journal of Biomechanics 41 (2), 399 (2008).

4 A. Ramuzat and M. L. Riethmuller, presented at the 11th Int. Symp. on Appl. of Laser Techniques to Fluid Flows, 2002 (unpublished).

5 S. Grosse, W. Schroder, and M. Klaas, Particle Image Velocimetry: New Developments and Recent Applications 112, 35 (2008).

6 S. K. Kim and S. K. Chung, Journal of Visualization 12 (3), 259 (2009).

7 A. Johnstone, M. Uddin, A. Pollard, A. Heenan, and W. Finlay, Experiments in Fluids 37 (5), 673 (2004).

8 Z. Zhang and C. Kleinstreuer, Journal of Aerosol Science 42 (3), 174 (2011).

9 C. L. Lin, M. H. Tawhai, G. McLennan, and E. A. Hoffman, Respiratory Physiology \& Neurobiology 157 (2-3), 295 (2007).

10 C. Kleinstreuer and Z. Zhang, International Journal of Multiphase Flow 29 (2), 271 (2003).

11 F. Menter, R. Langtry, S. Likki, Y. Suzen, P. Huang, and S. Volker, Journal of TurbomachineryTransactions of the Asme 128 (3), 413 (2006).

12 R. Theunissen and M. L. Riethmuller, Particle Image Velocimetry: New Developments and Recent Applications 112, 73 (2008). 